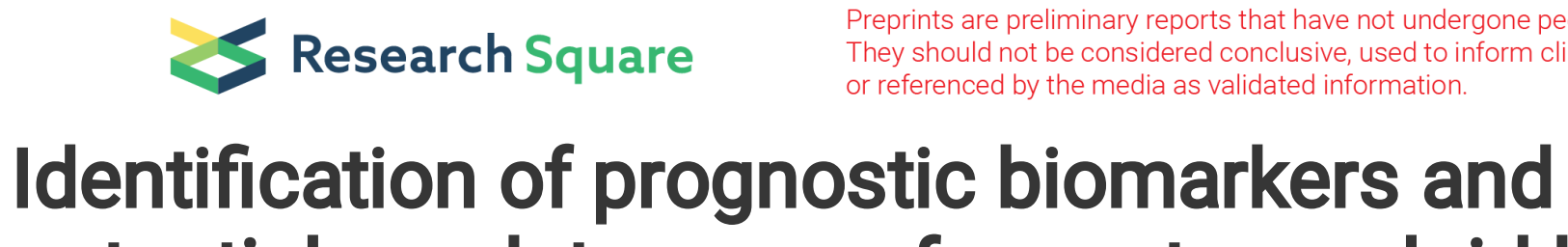 \\ potential regulatory axes for acute myeloid leukemia based on a competitive endogenous RNA network
}

\section{Mingde Li}

Anhui Medical University https://orcid.org/0000-0002-3072-4881

\section{Ying Chen}

AHUTCM: Anhui University of Traditional Chinese Medicine

\section{Zhaowu Chen}

The First Affiliated Hospital of USTC: Anhui Provincial Hospital

\section{Ming Chen}

Anhui Medical University

\section{Xingxing Huo}

AHUTCM: Anhui University of Traditional Chinese Medicine

SuBu ( $\sim$ busu0520@163.com )

AHUTCM: Anhui University of Traditional Chinese Medicine

\section{Research}

Keywords: acute myeloid leukemia, bioinformatics analysis, competitive endogenous RNA, prognostic biomarker, hsa-mir-206, NFAT5

Posted Date: February 19th, 2021

DOl: https://doi.org/10.21203/rs.3.rs-206813/v1

License: (9) This work is licensed under a Creative Commons Attribution 4.0 International License. Read Full License 


\section{Abstract \\ Background}

Acute myeloid leukemia (AML) is a common heterogeneous hematological malignancy with unclear pathogenesis and high mortality. Non-coding RNAs have recently received extensive attention. This study explored the potential mechanisms of interaction during the development of AML by analyzing a competitive endogenous RNA (ceRNA) network.

\section{Methods}

To obtain differentially expressed genes (DEGs), the transcripts and clinical AML data were downloaded from The Cancer Genome Atlas (TCGA) database. Bioinformatic analysis was used to predict the function annotation and RNA interaction. The data were used to construct a ceRNA regulatory network and survival analysis was used to discover key genes and predict potential regulatory axes. Quantitative Real-time PCR (qRT-PCR) was used to detect DEGs in HL-60 and THP-1 cell lines to verify the prediction.

\section{Results}

A ceRNA regulatory AML network with 164 IncRNA-miRNA-mRNA regulatory axes was constructed and visualized by Cytoscape software. Six IncRNAs were screened as potential prognostic factors for AML by survival analysis. Additionally, hsa-mir-206 and NFAT5 were discovered as key nodes in the ceRNA network. A CTB-193M12.1/hsa-mir-206/NFAT5 axis that was consistent with the ceRNA theory was identified. The qRT-PCR result showed that, compared with the normal control group, the expression of hsa-mir-206 in HL-60 and THP-1 cells was significantly decreased, while that of NFAT5 was moderately increased.

\section{Conclusions}

Therefore, the obtained ceRNA network and the CTB-193M12.1/hsa-mir-206/NFAT5 axis here may provide new view for exploring the pathogenic mechanisms of AML. NFAT5 and hsa-mir-206 were novel clinical predictors that may become key genes in AML.

\section{Introduction}

Acute myeloid leukemia (AML) is an aggressive hematological malignancy with a complex heterogeneous background, characterized by rapid proliferation of immature myeloid leukemia cells [1, 2]. The prognosis of AML is poor with its complex pathogenesis, which is not completely clear until now. Therefore, identification of related genes and clarification of pathogenesis and potential therapeutic targets of AML is critical. 
Long non-coding RNA (IncRNA) is a series of non-coding RNA with a length of more than 200 nucleotides [3]. Increasing number of studies have shown that these transcription "noises", which were previously regarded as having no biological function, play critical roles in several biological activities of eukaryotes, such as chromatin modification, post-transcriptional processing and nuclear transport[3-5]. MicroRNA (miRNA), a class of endogenous non-coding RNAs, maintain the balance of gene regulatory networks by binding to specific 3'UTR sites of messenger RNA (mRNA) [6]. It has been demonstrated that miRNAs play an important role in the pathogenesis of many diseases [7-9]. In addition, the ceRNA hypothesis suggests that the miRNA response element can act as a sponge for competitive binding of different types of RNA molecules, including IncRNA and mRNA, to regulate their interactions.

Recently, high-throughput sequencing technology has been widely used to identify cancer-related genes, providing a new perspective for exploring the genetic mechanisms of cancer [10]. The competitive endogenous RNA (ceRNA) mechanism proposed by Salmena et al. [11] suggests that transcription products such as IncRNA and mRNA can be used as ceRNAs to regulate gene expression levels and affect cell function by competing with miRNAs. Subsequently, several studies have reported that IncRNA, as a molecular sponge of miRNA, regulates mRNA expression and plays a role in tumorigenesis. For example, LncRNA SMAD5-AS1 can upregulate APC expression through sponging miR-135B-5p and inhibit proliferation of diffuse large B-cell lymphoma through the Wnt/ $\beta$-catenin pathway [12]. Lnc-SNHG1 can promote the progression of non-small cell lung cancer by acting as a sponge of miR-497 [13]. Therefore, the discovery of the IncRNA-miRNA-mRNA network may provide a new language for RNA communication and a comprehensive understanding of the etiology and pathogenesis of cancer.

In this study, through comprehensively integrating the gene and RNA expression data of AML, differentially expressed genes (DEGs) were screened out and the AML-related ceRNA regulatory network was established. Six genes significantly associated with survival were identified. Finally, we predicted the CTB-193M12.1/hsa-mir-206/NFAT5 regulatory axis. Experimental verification was performed to validate the key genes. This study may increase understanding of the molecular mechanism and provide new therapeutic concepts for AML.

\section{Materials And Methods}

\section{The Cancer Genome Atlas (TCGA) data retrieval}

The transcriptome datasets of $324 \mathrm{AML}$ patients and 150 normal subjects were retrieved from the TCGA database. Data types for AML patients and normal subjects included read counts of Gene Expression Quantification, FPKM, and Transcriptome Profiling miRNA Expression Quantification. The TCGA database includes standardized RNA expression profile data. The Ensemble official website was applied to annotate and separate IncRNA, miRNA, and mRNA, and the controversial RNAs were subsequently discarded.

\section{Identification of DEGs}


The transcriptome and miRNA data from the TCGA dataset were combined into a matrix. The differences of RNAs in AML were analyzed using the edgeR software package, which is based on the R language. A $P$-value lower than $0.01(P<0.01)$ and a fold change lower than 2 were used as thresholds to filter and screen differential genes. The "ggplot2" and "pheatmap" packages in the R software were used to generate volcano maps and thermal maps, respectively. This was done to demonstrate the differential expression of three gene sets.

\section{Functional annotation}

To elucidate the biological characteristics and processes of DEGs, Gene Ontology (GO) annotation and Kyoto Encyclopedia of Genes and Genomes (KEGG) pathway were analyzed via the Over-Representation method of the "clusterProfiler" package in R software [14]. The hypergeometric test method was adopted with a $\mathrm{P}<0.05$ as the threshold; the functional pathways that met the conditions were then sequenced. Afterward, the 10 significant biological pathway results were visualized.

\section{Construction of a ceRNA network}

First, miRcode [15] (http://www.mircode.org/) was used to retrieve the names of differentially expressed IncRNAs and predict their target miRNAs. The intersection of the target miRNAs and differentially expressed miRNAs was plotted. After eliminating the positive correlation pairs, the interacting IncRNAmiRNA regulatory axis was predicted. Subsequently, TargetScan (http://www.targetscan.org/) [16], miRDB (http://www.mirdb.org/) [17], and miRTarBase (http://mirtarbase.mbc.nctu.edu.tw/) [18] were used to predict the target mRNAs of differentially expressed miRNAs. MRNAs that did not coincide with differentially expressed mRNAs in the predicted result were discarded. After eliminating the positive correlation IncRNA-miRNA pairs, the target mRNAs were subsequently intersected with the differentially expressed mRNAs to construct the miRNA-mRNA regulatory axes. Finally, by combining the IncRNAmiRNA interactomes and the miRNA-mRNA interactomes, the ceRNA regulatory network was constructed (Figure 1). The Cytoscape software (v3.7.2) [19] was applied to visualize the constructed ceRNA network. The "cytoHubba" plugin was used to identify the central genes and their sub-network. The R package "ggalluvial" was used to confirm the ceRNA axes.

\section{Survival and prognosis analysis}

TCGA provided clinical information and survival data for patients with AML. The data were analyzed with the "survival" package in the R software. The "survminer" package was used for determining the most suitable cut-off value of the gene expression levels in the survival analysis. $P<0.05$ was significantly. Differential genes with a significant impact on survival were screened. Moreover, the GEPIA website was used to query the expression of DEGs, the relationship between different IncRNAs and NFAT5 was analyzed using R software.

\section{Cell Culture}


THP-1 and HL-60 cells were generously gifted by Professor Wenjiao Chang from the First Affiliated Hospital of China University of Science and Technology. Cells were maintained in RPMI1640 medium containing $10 \%$ heat-inactivated fetal bovine serum at $37^{\circ} \mathrm{C}$ in a $5 \% \mathrm{CO} 2$ atmosphere. The inoculation concentration was $2-3 \times 10^{5} \mathrm{celll} / \mathrm{ml}$, which was transmitted every $2-3 \mathrm{~d}$ to maintain logarithmic growth. Human peripheral blood mononuclear cells (PBMCs) from 15 normal persons were collected from the Health Examination Center of the First Affiliated Hospital of Anhui University of Traditional Chinese Medicine as the control group $[20,21]$. Written informed consent was obtained from all patients. This study was approved by the Hospital Ethics Committee.

\section{Quantitative Real-Time PCR (qRT-PCR)}

Total RNA was extracted from HL-60 cells, THP-1 cells, and normal human PBMCs using EZ-10 Total RNA Mini-Preps Kit (Sangon Biotech $₫$ Shanghai) according to the manufacturer's protocol. RiboSCRIPT ${ }^{\mathrm{TM}}$ mRNA/IncRNA qRT-PCR Starter Kit (Ribo Biotechnology, China) and Mir-X miRNA First-Strand Synthesis

Kit (Takara Biotechnology, China) was used for reverse transcription according to the manufacturer's protocol. TB Green ${ }^{\circledR}$ Premix Ex Taq ${ }^{\text {TM }}$ II (Tli RNaseH Plus) (Takara Biotechnology, China) was used for qRT-PCR. Roche LightCycler ${ }^{\circledR} 480$ II Real-time PCR Systems (Roche Diagnostics Ltd.) was used for qRTPCR assay. GAPDH was used as an internal reference for IncRNA and mRNA expression, and U6 was used to normalize miRNA expression. The thermocycling conditions were as follows: $95^{\circ} \mathrm{C}$ for $10 \mathrm{~min}$, followed by 40 cycles of $95^{\circ} \mathrm{C}$ for $5 \mathrm{sec}, 60^{\circ} \mathrm{C}$ for $30 \mathrm{sec}$ and $72^{\circ} \mathrm{C}$ for $30 \mathrm{sec}$. Sequences for primers were as follows: hsa-miR-206 forward, 5'-ACACTCCAGCTGGGTGGAATGTAAGGAAGT-3' and reverse, 5'TGGTGTCGTGGAGTCG-3', NFAT5 forward, 5'-TAATGCCCTGATGACTCCAC-3' and reverse, 5'CCATTTCCTGCTATGTTTGAG-3', GAPDH forward, 5'-CGAGCCACATCGCTCAGACA-3' and reverse, 5'GTGGTGAAGACGCCAGTGGA-3', U6 forward, 5'- CTCGCTTCGGCAGCACA-3' and reverse, 5'AACGCTTCACGAATTTTCGT-3'. All primers used for amplification of targets were designed and synthesized by Sangon Biotech. The analysis of relative expression levels was executed by the $2^{-\Delta \Delta C t}$ method [22].

\section{Results}

\section{DEGs in AML}

A total of 7897 differently expressed IncRNAs, 430 differentially expressed miRNAs, and 10766 differentially expressed mRNAs were filtered, of which 5115 (64.8\%) IncRNAs, 170 miRNAs (42.2\%), and 3461 (32.1\%) mRNAs were upregulated. Additionally, 2782 (35.2\%) IncRNAs, 233 miRNAs (57.8\%), and 7305 (67.9\%) mRNAs were downregulated in AML. The volcanic plots were produced to observe RNA expression profiles (Figure 2). Cluster analysis was performed according to the expression of three types of differential genes, and the results were depicted via the "pheatmap" package in the R software (Figure $3)$.

\section{GO and KEGG pathway analysis of differently expressed mRNAs}


The biological characteristics and functional annotations of differentially expressed mRNAs were described by enriching on $\mathrm{GO}$ and KEGG, respectively. $\mathrm{GO}$ enrichment analysis indicated that differentially expressed mRNAs were significantly enriched in extracellular structure organization, extracellular matrix and receptor regulator activity (Figure 4a). Moreover, KEGG enrichment analysis revealed that multiple enrichment pathways, such as neuroactive ligand-receptor interaction, cytokine-cytokine receptor interaction, and axon guidance (Figure $4 b$ ).

\section{Construction of a ceRNA network in AML}

A total of 720 potential IncRNA-miRNA regulatory axes were obtained by intersecting the screened differentially expressed miRNAs with the predicted results. After screening the predicted results, 1535, 21068, and 2031 regulatory axes were found in TargetScan, miRDB, and miRTarBase, respectively. The acquired results were intersected to obtain 37 high confidence miRNA-mRNA regulatory axes. Finally, the ceRNA regulatory network was constructed with 164 IncRNA-miRNA-mRNA axes and the RNA interaction relationships in AML were predicted (Figure 5). Sanky diagram drawn by "ggalluvial" package in $\mathrm{R}$ software was used to show the regulatory relationship of ceRNA (Figure 6).

\section{Open in a separate windowPrognostic significance of RNAs in the ceRNA network}

The survival analysis indicated that the expression of six IncRNAs, including ENSG00000242767 (ZBTB20-AS4), ENSG00000227409 (ZMYM4-AS1), ENSG00000253539 (RP11-402L5.1), ENSG00000257769 (CTB-193M12.1), ENSG00000232712 (KIZ-AS1), and ENSG00000248734 (CTD2260A17.1), were significantly related to the survival rate of the patients $(p<0.05)$ (Figure 7). Thus, the survival-related IncRNA-miRNA-mRNA regulatory axes were obtained by searching the target genes of IncRNAs in the ceRNA regulatory network. Notably, among the aforementioned regulatory axes, a strong correlation was found between CTB-193M12.1 and NFAT5, which provided a direction for the next prediction of potential ceRNA regulatory axes.

\section{Identification of a potential regulatory axis}

CTB-193M12.1/hsa-mir-206/NFAT5 was predicted as a potential regulatory axis of the ceRNA network for the following reasons: analysis in the GEPIA website showed that CTB-193M12.1 and NFAT5 is a luxury gene specifically expressed in AML patients (Figure 8a, b). A total of 31 tumor types with specific expression of this gene were identified by the GEPIA website, and the conclusion showed that CTB193M12.1 is specifically overexpressed in AML patients. Then, the interaction between CTB-193M12.1 and hsa-mir-206 was demonstrated using an extremely dependable database (LncBase). Similarly, a total of three highly reliable databases (miRTarBase, miRDB, and TargetScan) were used to predict the interaction between hsa-mir-206 and NFAT5. Furthermore, survival analysis proved that the high expression level of CTB-193M12.1 was significantly correlated with the low survival rate. And the expression level of NFAT5 was extremely high in AML patients (Figure 8b). Ultimately, the GEPIA website was used to determine the correlation between CTB-193M12.1 and NFAT5, and the obtained results were compared with the data analysis result. The outcome demonstrated that the expression of CTB- 
193M12.1 was correlated significantly with that of NFAT5 $(r=0.82, P<0.01)$, which was consistent with the correlation analysis result and the ceRNA theory (Figure 8c). In summary, the data suggested that CTB-193M12.1 and NFAT5 expression were significantly increased and positively correlated with each other, while hsa-mir-206 was significantly decreased in AML patients. These findings are consistent with the "ceRNA hypothesis", which suggests that IncRNAs indirectly regulate the expression of mRNAs via modulating the expression of miRNAs. Therefore, we conclude that the CTB-193M12.1/hsa-mir-206/ NFAT5 axis might become a potential regulatory axis of the ceRNA network and may have a profound effect on the progression of AML (Figure 9).

\section{Experimental validation}

The expression levels of hsa-mir-206 and NFAT5 in HL-60 cells, THP-1 cells, and 15 normal PBMC samples were detected by qRT-PCR. We discovered that the expression level of hsa-mir-206 was significantly decreased while the expression level of NFAT5 was moderately increased in HL-60 cells and THP-1 cells (Figure 10). The results of qRT-PCR validated the TCGA data and also provided basic evidence for our conjecture.

\section{Discussion}

It has been elucidated that several IncRNAs, miRNAs, and mRNAs are differentially expressed in AML, and the ceRNA network might be an instrumental knot in the regulation of tumorigenesis [23]. Therefore, it is urgent to identify key genes and understand the pathogenesis of AML. This study used a series of methods to identify and analyze the data downloaded from TCGA and GTEx databases and verify the obtained results.

Here, the differentially expressed genes were obtained by analyzing the downloaded data. GO and KEGG enrichment analysis revealed that differentially expressed RNAs and notable pathways of enrichment were all associated with the progression of AML. A ceRNA network constructed by 164 regulatory axes was screened. A total of six IncRNAs (ZBTB20-AS4, ZMYM4-AS1, RP11-402L5.1, CTB-193M12.1, KIZ-AS1, and CTD-2260A17.1) were identified from the constructed ceRNA network through survival analysis, which were significantly associated with overall survival rate $(P<0.05)$ (Fig. 7). Patients with high expression of CTB-193M12.1 indicated poor prognosis (Fig. 7a). The correlation analysis showed that the interaction between CTB-193M12.1 and NFAT5 was significantly strong $(r=0.82, P<0.01)$ (Fig. 8c). A potential CTB-193M12.1/hsa-mir-206/NFAT 5 regulatory axis with the highest correlation was constructed ultimately, which was basically in line with the ceRNA theory (Fig. 9). The results of qRT-PCR validated the differentially expressed of hsa-mir-206 and NFAT5 (Fig. 10). The analysis aimed to provide new ideas for a comprehensive and deeper understanding of the molecular mechanism of AML.

LncRNA, a functional RNA molecule that plays a role in various biological functions of the human body, contributes significantly to the aforementioned regulatory network. Since studies focused on IncRNAs are still limited, only three have official human genome nomenclature committee symbols (ZBTB20-AS4, ZMYM4-AS1, and KIZ-AS1) and none have been reported. However, cluster analysis showed that CTB- 
193M12.1 and NFAT5 expression were increased in AML patients while hsa-mir-206 was decreased (Fig. 3). Our bioinformatics analysis results also revealed a strong correlation between CTB-193M12.1 and AML (Fig. 7a). Besides, several studies indicate that abnormal expression of IncRNAs is often associated with the development of AML $[3,24]$. Therefore, it is reasonable to suggest CTB-193M12.1 as a regulatory gene of AML.

As a binding site for multiple RNAs, miRNA are also indispensable in tumorigenesis. Interestingly, our results revealed that of 163 IncRNA-miRNA-mRNA regulatory axes, 145 were regulated by hsa-mir-206, which indicated that hsa-mir-206 may be the strongest regulator in the pathogenesis of AML. Recently, studies on hsa-mir-206 have become a hot topic, but studies on AML are still lacking. Notably, we found that a recent study by Chen et al. [25] on occupational asthma (OA) proposed a regulatory axis similar to that in this study. They pointed out that miR-206-3p, as a key factor in calcineurin/NFAT signaling in macrophages and bronchoalveolar lavage cells, affects the transcription of iNOS and thus regulates the development of OA. This proven hsa-mir-206/NFAT regulatory axis correlates with our findings. Further, Chen et al. also used the THP- 1 cell line. Therefore, it is reasonable to suggest that hsa-mir-206 can act as a key site in regulating the development of AML by mediating the expression of NFAT5. Moreover, it has been reported that low expression of hsa-mir-206 is closely associated with the poor prognosis of pediatric AML patients [26]. This is consistent with our finding that the downregulation of hsa-mir-206 predicts poor prognosis in AML. It is noteworthy that our study was conducted in adults, which not only verified the above view but also provided powerful evidence that hsa-mir-206 may have an impact on the pathogenesis of AML. Also, hsa-mir-206 plays an important role in other cancers. For example, downregulation of miR-206 leads to poor prognosis in endometrial cancer [27] and promotes the occurrence and development of laryngeal cancer [28]. High expression of miR-206 inhibits osteosarcoma cell proliferation [29], as well as the proliferation and migration of prostate cancer cells [30]. Overall, these results suggested that hsa-mir-206 can regulate multiple cancer processes and might play an important role in AML through the hsa-mir-206/NFAT5 regulatory axis.

Similarly, NFAT5, which is a target gene of hsa-mir-206 in the ceRNA network, has been shown to regulate multiple tumor processes. For example, NFAT 5 can act as a ceRNA regulated by circFOXO3, plays a role in glioblastoma through sponging miR-138-5p and miR-432-5p [31], and may also regulate the progression of colon cancer through the MALAT1/miR1295p/NFAT5 axis [32]. Here, results from our investigation and the GEPIA website both showed that NFAT5 has a strong relationship with CTB193M12.1 (Fig. 8C) and that the low expression of NFAT5 can lead to a higher survival rate. Moreover, one study also found that the downregulation of NFAT5, which is regulated by an upstream gene, led to a more optimistic prognosis in lung cancer [33]. This demonstrates that high expression of NFAT5 can lead to poor prognosis in cancer and may also participate in the progression of multiple cancer types as a ceRNA. Therefore, it is reasonable to suggest that NFAT5 may regulate the progression of AML through the CTB-193M12.1/hsa-mir-206/NFAT5 ceRNA regulatory axis proposed in this study.

Recently, studies on the association of ceRNA with AML have emerged, but most of them only focused on the association between IncRNA dysregulation and AML [34,35]. Among a study aimed at pediatric AML, 
although the biomarkers based on the ceRNA network have been analyzed [36], whether the biomarkers suggested in the study applies to adult AML still need further validation. Another research proposed by Wang et al. [37] constructed a coexpression ceRNA network and identified several cancer-related genes, but the interactional relationships between differentially expressed genes still not been analyzed. Our current research aimed to establish a more integrated regulatory network and select the most relevant regulatory axis to an in-depth study, in order to identify several prognostic biomarkers and understand the new "language" of communication between RNA transcripts from multiple dimensions in AML.

However, there are still some limitations in our study. The interaction between RNAs has not been experimentally confirmed. It is necessary to create experiments to verify the CTB-193M12.1/hsa-mir206/NFAT5 regulatory axis in future studies.

\section{Conclusions}

In conclusion, a potential regulatory axis was constructed, which revealed the correlation between genes and may fulfill valuable functions in the biological process of AML. Several new prognostic and predictive biomarkers were identified, which may provide a new direction for revealing the pathogenesis of AML.

\section{Abbreviations}

AML: Acute myeloid leukemia; ceRNA: competitive endogenous RNA; DEGs: differentially expressed genes; TCGA: The Cancer Genome Atlas; qRT-PCR: Quantitative Real-time PCR; IncRNA: Long non-coding RNA; miRNA: MicroRNA; mRNA: messenger RNA; GO: Gene Ontology; KEGG: Kyoto Encyclopedia of Genes and Genomes; PBMCs: peripheral blood mononuclear cells;

\section{Declarations}

\section{Ethics approval and consent to participate}

Not applicable.

\section{Consent for publication}

The recruited personnel have signed written informed consent.

\section{Availability of data and materials}

The RNA and miRNA expression profiles and clinical follow-up data used during the present study are obtained from a public database of TCGA. Moreover, the datasets used to support the findings of this study are included within the article, and are available from the corresponding author upon reasonable request. 


\section{Competing interests}

The authors declare that they have no conflict of interest.

\section{Funding}

This study was supported by the National Natural Science Foundation of China (No. 81803938) and Anhui University of Traditional Chinese Medicine Natural Science Foundation (No. 2019zryb12, No. 2020yfyzc06).

\section{Authors' contributions}

Xingxing Huo conceived and designed the experiments and performed the experiments. Xingxing Huo, Su $\mathrm{Bu}$, Mingde Li, and Ying Chen analyzed the data. Xingxing Huo and Zhaowu Chen contributed reagents/materials/analysis tools. Mingde Li, Su Bu, and Ming Chen wrote the article. Xingxing Huo approved the final version.

\section{Acknowledgements}

Not applicable.

\section{References}

1. Pulikkan JA, Hegde M, Ahmad HM, Belaghzal H, Illendula A, Yu J, et al. CBFbeta-SMMHC Inhibition Triggers Apoptosis by Disrupting MYC Chromatin Dynamics in Acute Myeloid Leukemia. Cell. 2018; 174:172-86 e21.

2. Thomas D, Majeti R. Biology and relevance of human acute myeloid leukemia stem cells. Blood. 2017; 129:1577-85.

3. Feng Y, Hu S, Li L, Zhang S, Liu J, Xu X, et al. LncRNA NR-104098 Inhibits AML Proliferation and Induces Differentiation Through Repressing EZH2 Transcription by Interacting With E2F1. Front Cell Dev Biol. 2020; 8:142.

4. Wolter JM, Mao H, Fragola G, Simon JM, Krantz JL, Bazick HO, et al. Cas9 gene therapy for Angelman syndrome traps Ube3a-ATS long non-coding RNA. Nature. 2020; 587:281-4.

5. Yang T, Li H, Chen T, Ren H, Shi P, Chen M. LncRNA MALAT1 Depressed Chemo-Sensitivity of NSCLC Cells through Directly Functioning on miR-197-3p/p120 Catenin Axis. Mol Cells. 2019; 42:270-83.

6. Deng JH, Deng Q, Kuo CH, Delaney SW, Ying SY. MiRNA targets of prostate cancer. Methods Mol Biol. 2013; 936:357-69.

7. Pratama MY, Visintin A, Croce LS, Tiribelli C, Pascut D. Circulatory miRNA as a Biomarker for Therapy Response and Disease-Free Survival in Hepatocellular Carcinoma. Cancers (Basel). 2020; 12.

8. Deng S, Zhang X, Qin Y, Chen W, Fan H, Feng X, et al. miRNA-192 and -215 activate Wnt/beta-catenin signaling pathway in gastric cancer via APC. J Cell Physiol. 2020; 235:6218-29. 
9. Zhao J, Zhu XC, Wu XS, Wang L, Zhu CC, Yang K, et al. Identification of miR-4644 as a suitable endogenous normalizer for circulating miRNA quantification in hepatocellular carcinoma. $\mathrm{J}$ Cancer. 2020; 11:7032-44.

10. Mounir M, Lucchetta M, Silva TC, Olsen C, Bontempi G, Chen X, et al. New functionalities in the TCGAbiolinks package for the study and integration of cancer data from GDC and GTEx. PLoS Comput Biol. 2019; 15:e1006701.

11. Salmena L, Poliseno L, Tay Y, Kats L, Pandolfi PP. A ceRNA hypothesis: the Rosetta Stone of a hidden RNA language? Cell. 2011; 146:353-8.

12. Zhao CC, Jiao Y, Zhang YY, Ning J, Zhang YR, Xu J, et al. Lnc SMAD5-AS1 as ceRNA inhibit proliferation of diffuse large $B$ cell lymphoma via Wnt/beta-catenin pathway by sponging miR-135b$5 p$ to elevate expression of APC. Cell Death Dis. 2019; 10:252.

13. Li Z, Lu Q, Zhu D, Han Y, Zhou X, Ren T. Lnc-SNHG1 may promote the progression of non-small cell lung cancer by acting as a sponge of miR-497. Biochem Biophys Res Commun. 2018; 506:632-40.

14. Yu G, Wang LG, Han Y, He QY. clusterProfiler: an R package for comparing biological themes among gene clusters. OMICS. 2012; 16:284-7.

15. Jeggari A, Marks DS, Larsson E. miRcode: a map of putative microRNA target sites in the long noncoding transcriptome. Bioinformatics. 2012; 28:2062-3.

16. Agarwal V, Bell GW, Nam JW, Bartel DP. Predicting effective microRNA target sites in mammalian mRNAs. Elife. 2015; 4.

17. Wong N, Wang X. miRDB: an online resource for microRNA target prediction and functional annotations. Nucleic Acids Res. 2015; 43:D146-52.

18. Chou CH, Shrestha S, Yang CD, Chang NW, Lin YL, Liao KW, et al. miRTarBase update 2018: a resource for experimentally validated microRNA-target interactions. Nucleic Acids Res. 2018; 46:D296-D302.

19. Shannon P, Markiel A, Ozier O, Baliga NS, Wang JT, Ramage D, et al. Cytoscape: a software environment for integrated models of biomolecular interaction networks. Genome Res. 2003; 13:2498-504.

20. He L, Shi L, Du Z, Huang H, Gong R, Ma L, et al. Mebendazole exhibits potent anti-leukemia activity on acute myeloid leukemia. Exp Cell Res. 2018; 369:61-8.

21. Herz C, Tran HTT, Landerer S, Gaus J, Schlotz N, Lehr L, et al. Normal human immune cells are sensitive to telomerase inhibition by Brassica-derived 3,3-diindolylmethane,partly mediated via ERalpha/beta-AP1 signaling. Mol Nutr Food Res. 2017; 61.

22. Livak KJ, Schmittgen TD. Analysis of relative gene expression data using real-time quantitative PCR and the 2(-Delta Delta C(T)) Method. Methods. 2001; 25:402-8.

23. Sanchez-Mejias A, Tay Y. Competing endogenous RNA networks: tying the essential knots for cancer biology and therapeutics. J Hematol Oncol. 2015; 8:30. 
24. Liang Y, Li E, Zhang H, Zhang L, Tang Y, Wanyan Y. Silencing of IncRNA UCA1 curbs proliferation and accelerates apoptosis by repressing SIRT1 signals by targeting miR-204 in pediatric AML. J Biochem Mol Toxicol. 2020; 34:e22435.

25. Lin CC, Law BF, Hettick JM. Acute 4,4'-Methylene Diphenyl Diisocyanate Exposure-Mediated Downregulation of miR-206-3p and miR-381-3p Activates Inducible Nitric Oxide Synthase Transcription by Targeting Calcineurin/NFAT Signaling in Macrophages. Toxicol Sci. 2020; 173:10013.

26. Liu H, Wu H, Qin X. MicroRNA-206 serves as a tumor suppressor in pediatric acute myeloid leukemia by targeting Cyclin D1. Pathol Res Pract. 2019; 215:152554.

27. Zheng Y, Yang X, Wang C, Zhang S, Wang Z, Li M, et al. HDAC6, modulated by miR-206, promotes endometrial cancer progression through the PTEN/AKT/mTOR pathway. Sci Rep. 2020; 10:3576.

28. Zhang T, Liu M, Wang C, Lin C, Sun Y, Jin D. Down-regulation of MiR-206 promotes proliferation and invasion of laryngeal cancer by regulating VEGF expression. Anticancer Res. 2011; 31:3859-63.

29. Xu X, Qiu B, Yi P, Li H. Overexpression of miR-206 in osteosarcoma and its associated molecular mechanisms as assessed through TCGA and GEO databases. Oncol Lett. 2020; 19:1751-8.

30. Wang Y, Xu H, Si L, Li Q, Zhu X, Yu T, et al. MiR-206 inhibits proliferation and migration of prostate cancer cells by targeting CXCL11. Prostate. 2018; 78:479-90.

31. Zhang S, Liao K, Miao Z, Wang Q, Miao Y, Guo Z, et al. CircFOXO3 promotes glioblastoma progression by acting as a competing endogenous RNA for NFAT5. Neuro Oncol. 2019; 21:1284-96.

32. Hu D, Zhang B, Yu M, Shi W, Zhang L. Identification of prognostic biomarkers and drug target prediction for colon cancer according to a competitive endogenous RNA network. Mol Med Rep. 2020; 22:620-32.

33. Meng X, Li Z, Zhou S, Xiao S, Yu P. miR-194 suppresses high glucose-induced non-small cell lung cancer cell progression by targeting NFAT5. Thorac Cancer. 2019; 10:1051-9.

34. Heitmann JS, Hagelstein I, Hinterleitner C, Roerden M, Jung G, Salih HR, et al. Identification of CD318 (CDCP1) as novel prognostic marker in AML. Ann Hematol. 2020; 99:477-86.

35. Feng S, Liu N, Chen X, Liu Y, An J. Long non-coding RNA NEAT1/miR-338-3p axis impedes the progression of acute myeloid leukemia via regulating CREBRF. Cancer Cell Int. 2020; 20:112.

36. Zhang Y, Liu Y, Xu X. Knockdown of LncRNA-UCA1 suppresses chemoresistance of pediatric AML by inhibiting glycolysis through the microRNA-125a/hexokinase 2 pathway. J Cell Biochem. 2018; 119:6296-308.

37. Wang JD, Zhou HS, Tu XX, He Y, Liu QF, Liu Q, et al. Prediction of competing endogenous RNA coexpression network as prognostic markers in AML. Aging (Albany NY). 2019; 11:3333-47.

\section{Figures}




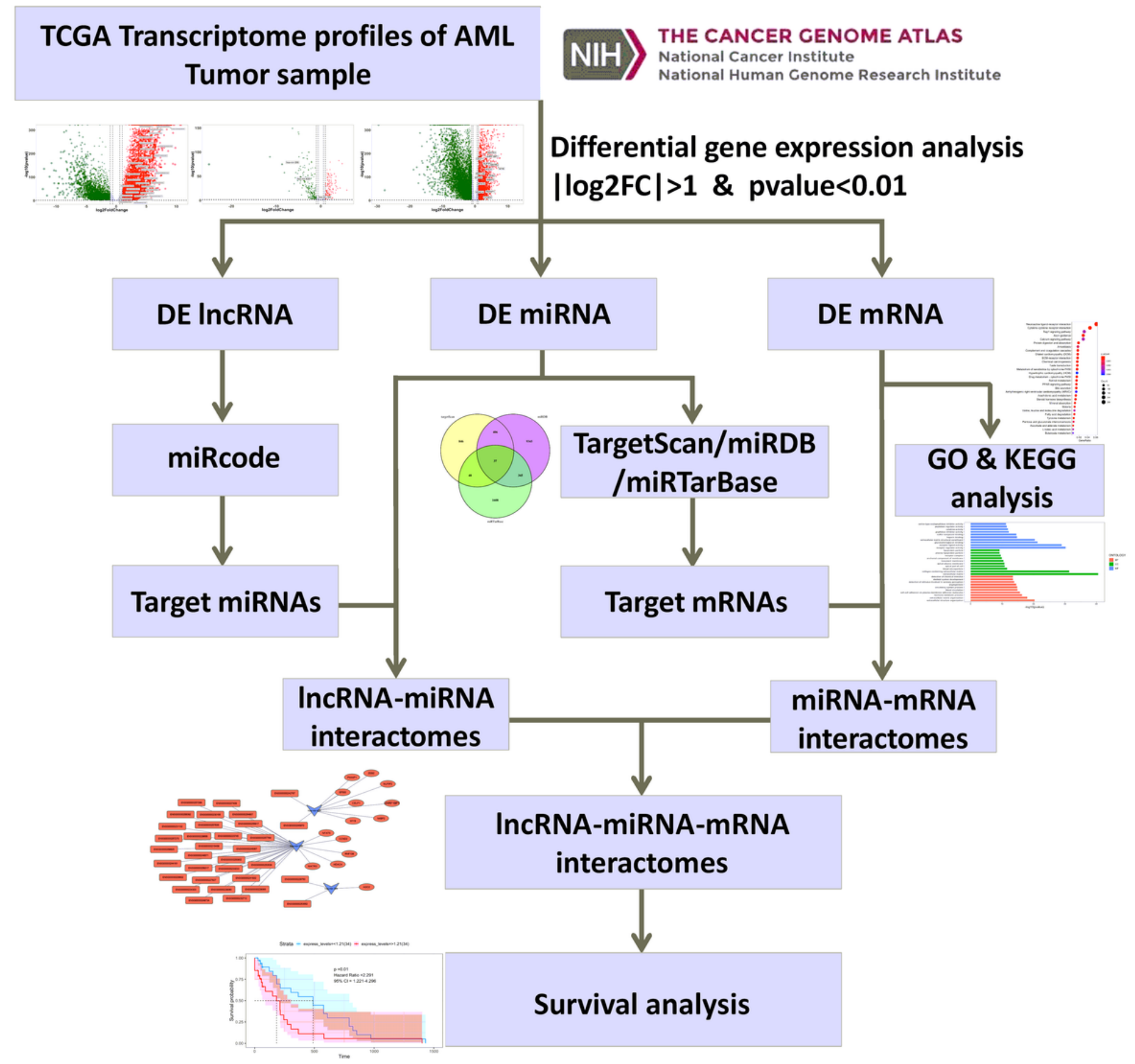

Figure 1

Construction flow-process diagram of the ceRNA network in AML. 


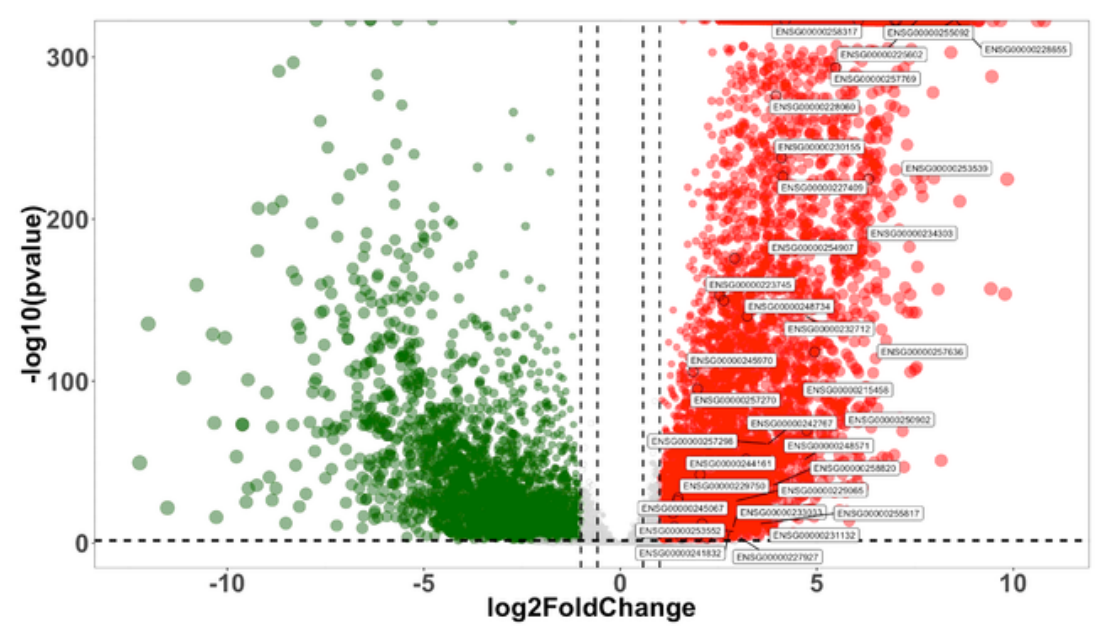

(a)

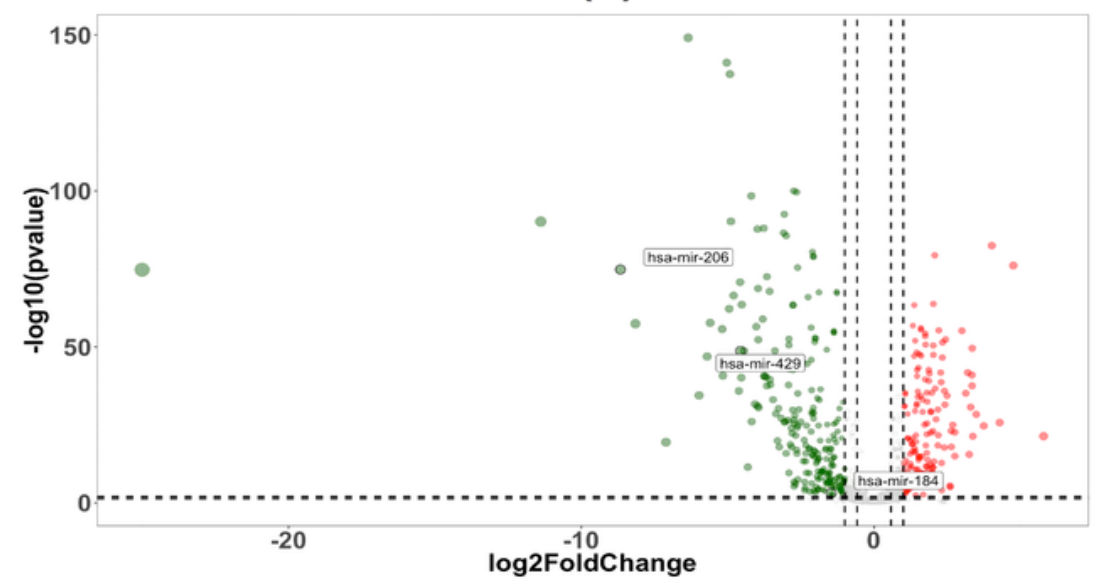

(b)

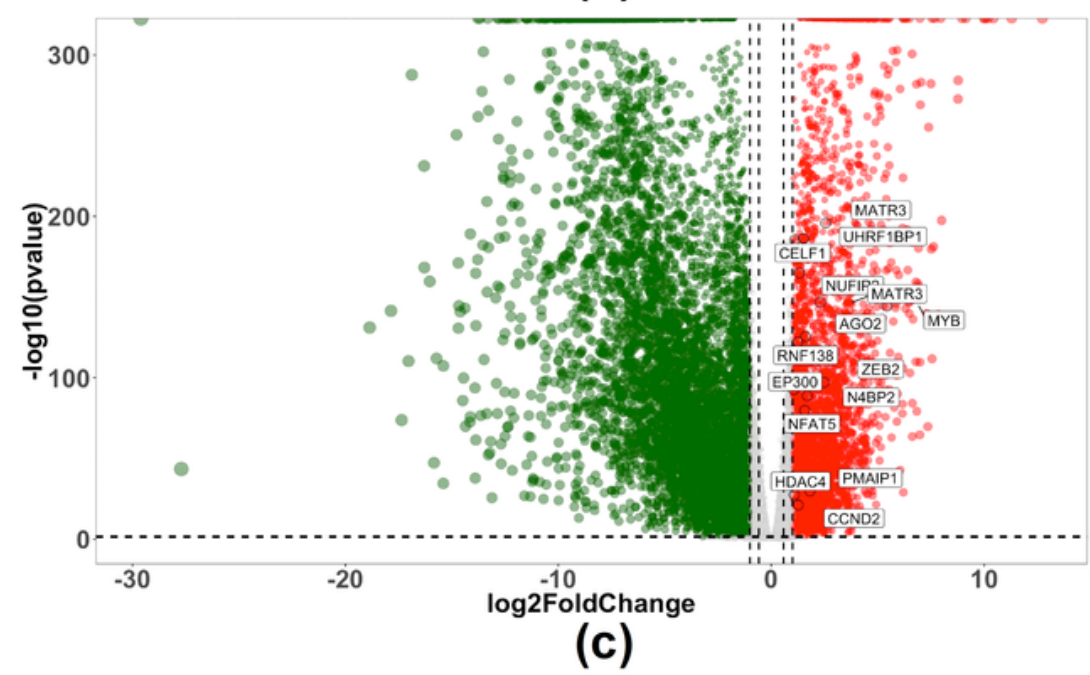

\section{Figure 2}

Screening differentially expressed genes (DEGs). Volcano diagrams of AML-related differentially expressed (a) IncRNAs, (b) miRNAs, and (c) mRNAs. The abscissa represents the logFC, and the ordinate represents the P-value. The red dots indicate the upregulated genes and the green dots indicate the downregulated genes with significant differences. 


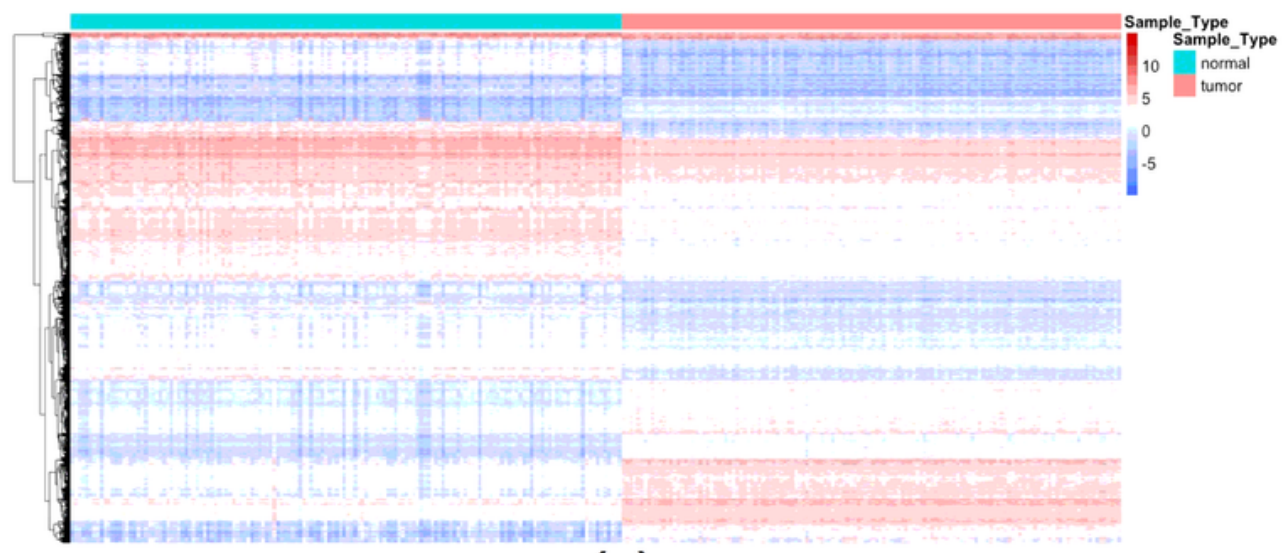

(a)

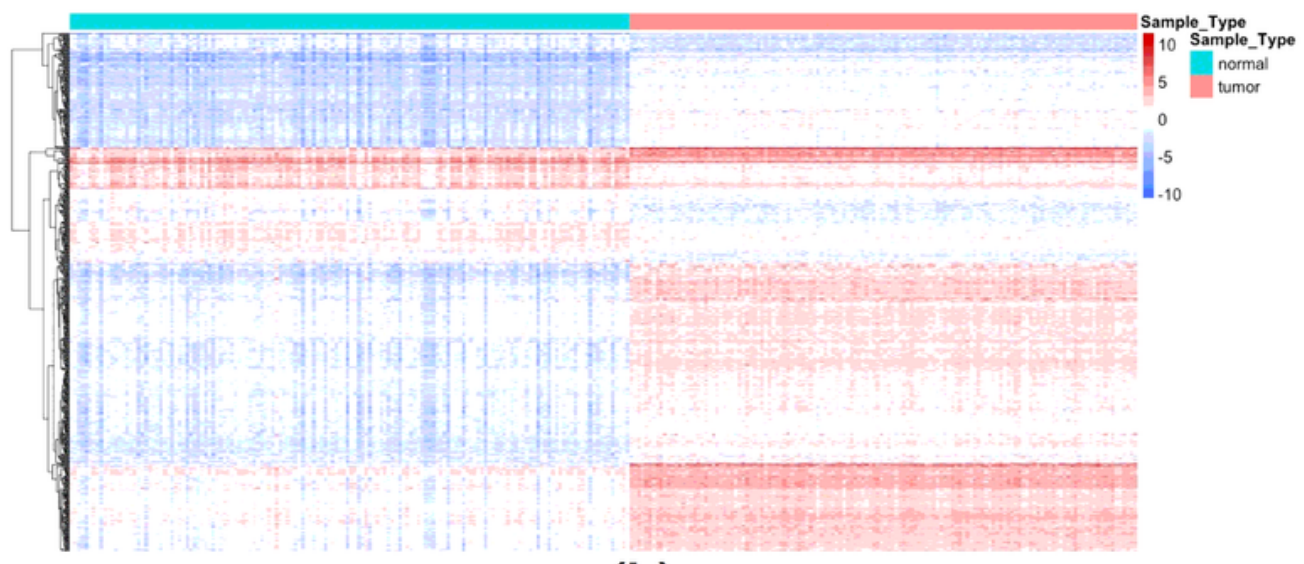

(b)

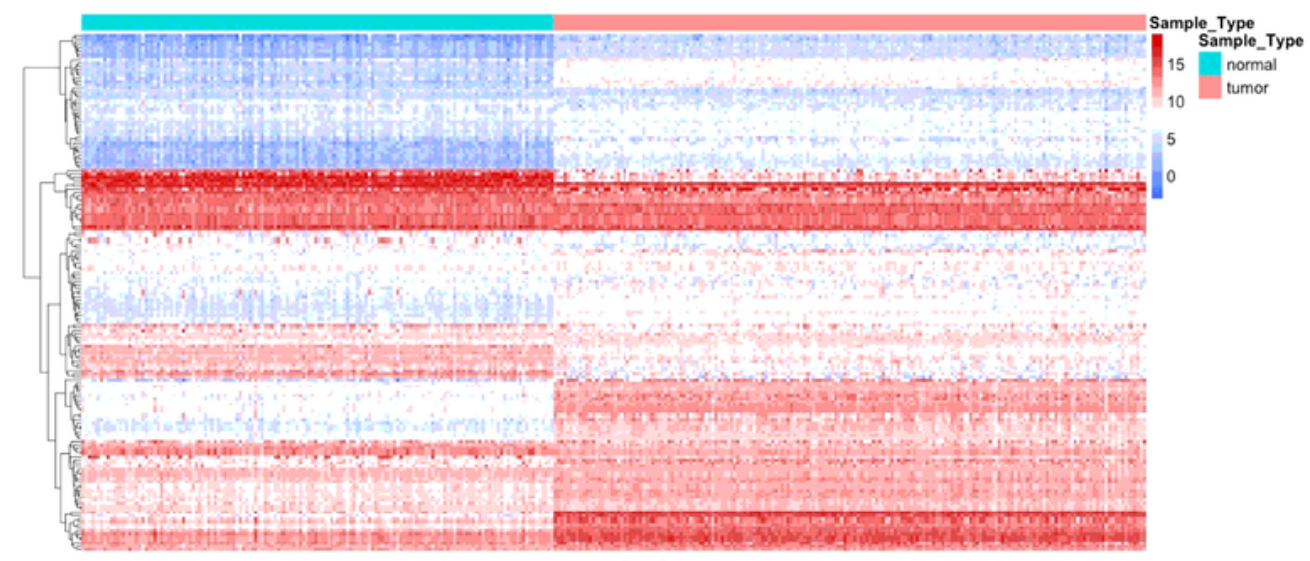

(c)

\section{Figure 3}

Cluster analysis of the expression levels of the correlated genes in the ceRNA regulatory network. Cluster thermogram of expression levels of (a) IncRNAs, (b) miRNAs, and (c) mRNAs in the ceRNA regulatory network. Blue indicates downregulation and red indicates upregulation. Red in the annotation axis indicates disease samples, while blue indicates normal samples. 


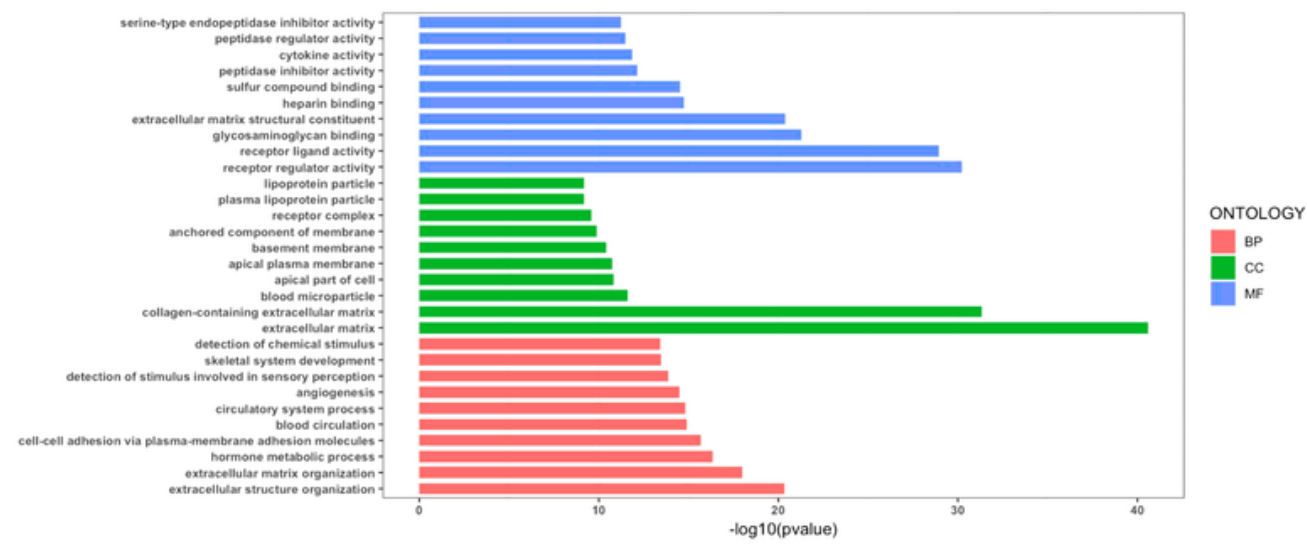

(a)

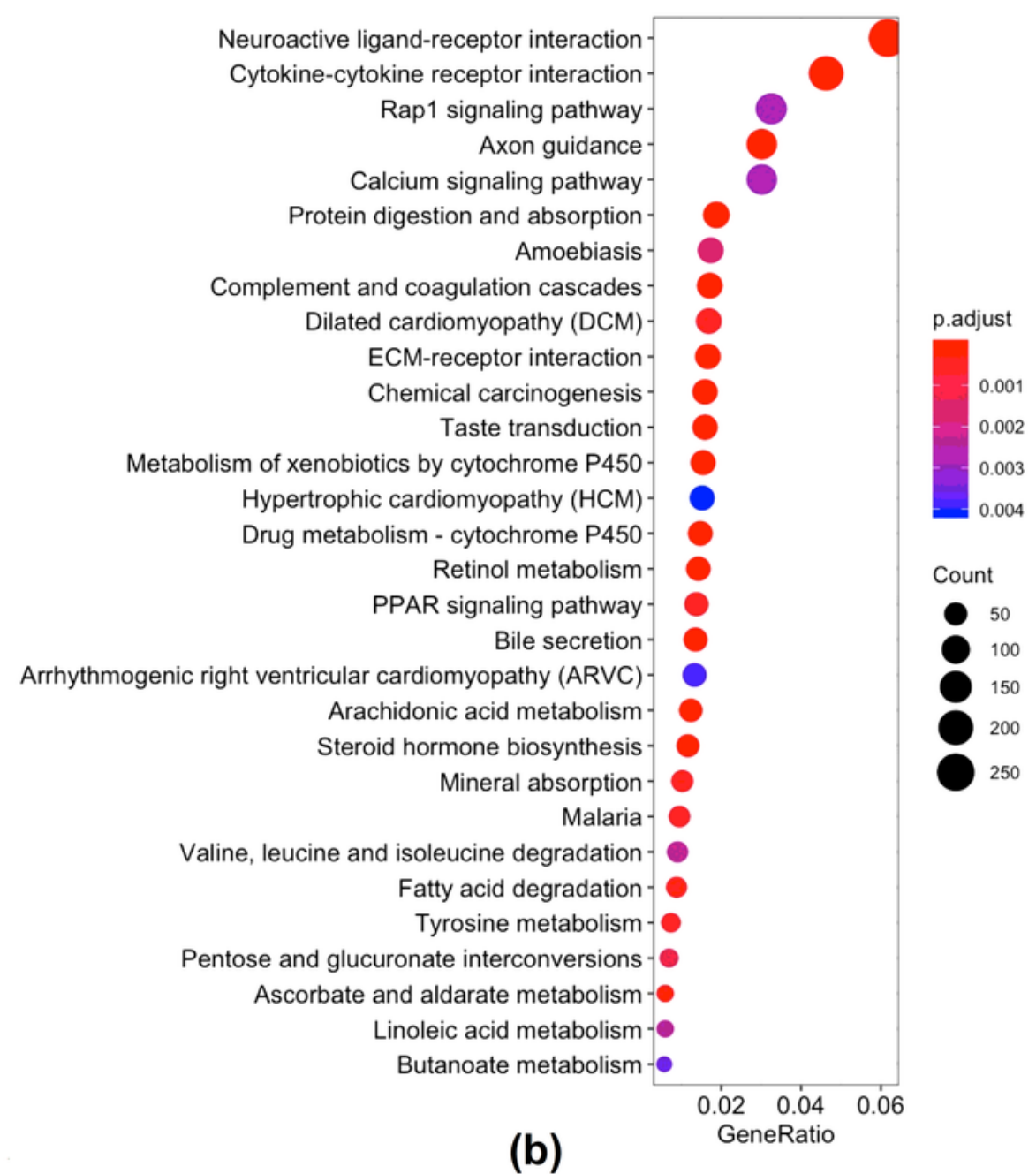

\section{Figure 4}

Functional enrichment analyses of the differentially expressed RNAs. (a) The significantly enriched GO biological process and the relevant genes; (b) The significantly enriched KEGG pathways and the relevant genes. 


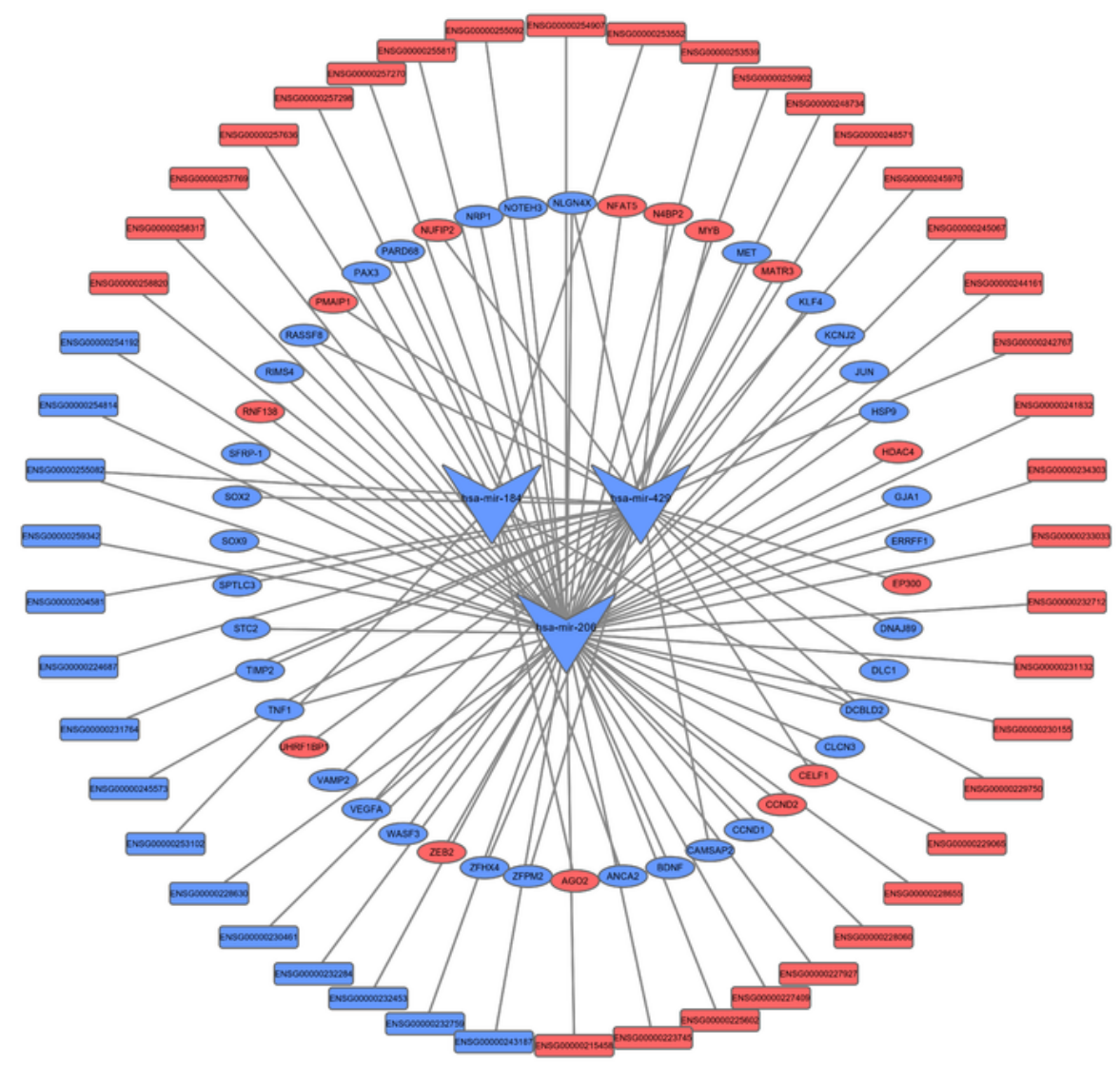

(a)

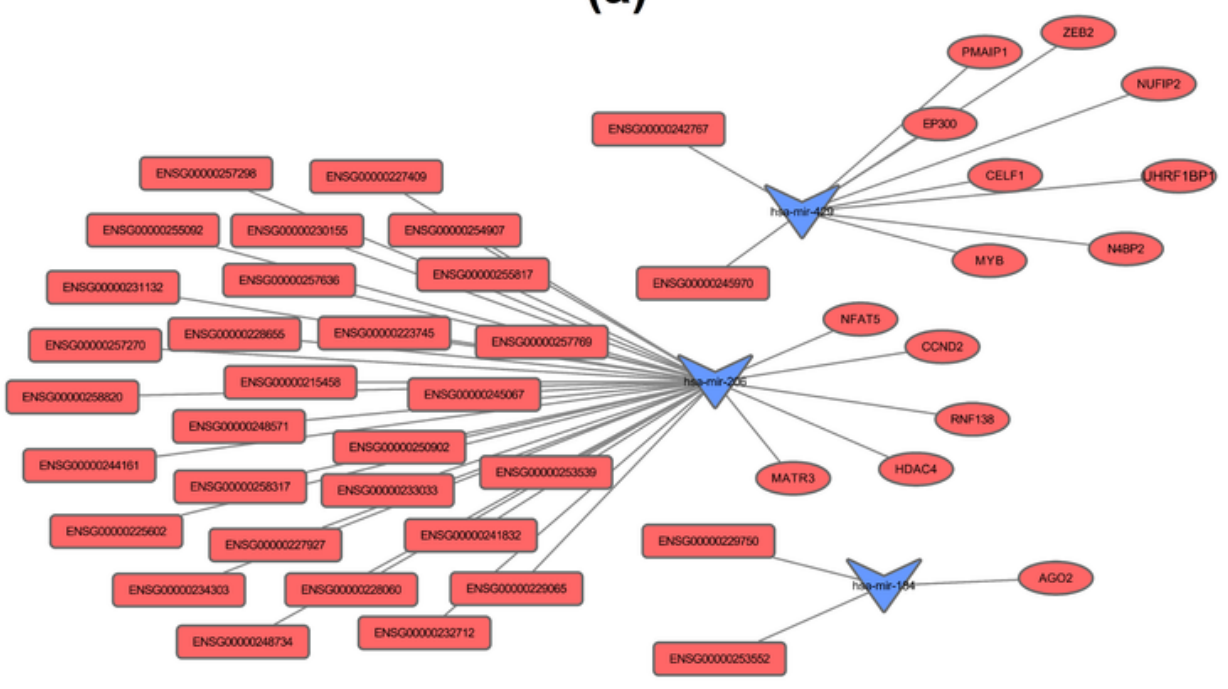

(b)

\section{Figure 5}

Construct ceRNA regulatory network and axes in AML. (a) A comprehensive view on the regulatory network of the ceRNA in AML. (b) A comprehensive view on the regulatory axes of the ceRNA in AML. Red indicates the differential upregulation of genes, while blue indicates the differential downregulation. LncRNA, miRNA, and mRNA are represented by rectangle, $V$, and ellipse shapes, respectively. 


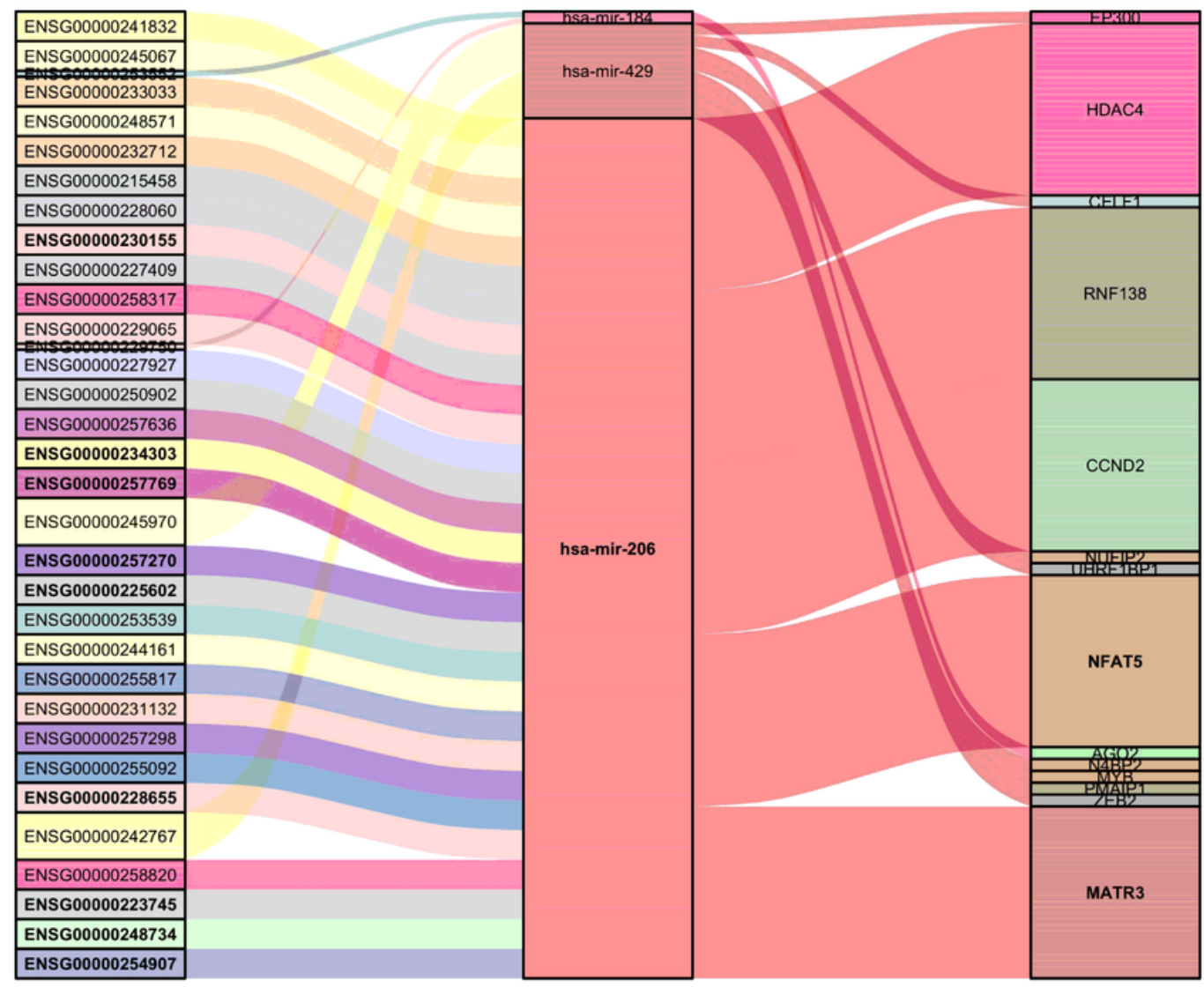

Figure 6

Sankey diagram was used for displaying the IncRNA-miRNA-mRNA regulatory axes in AML. 


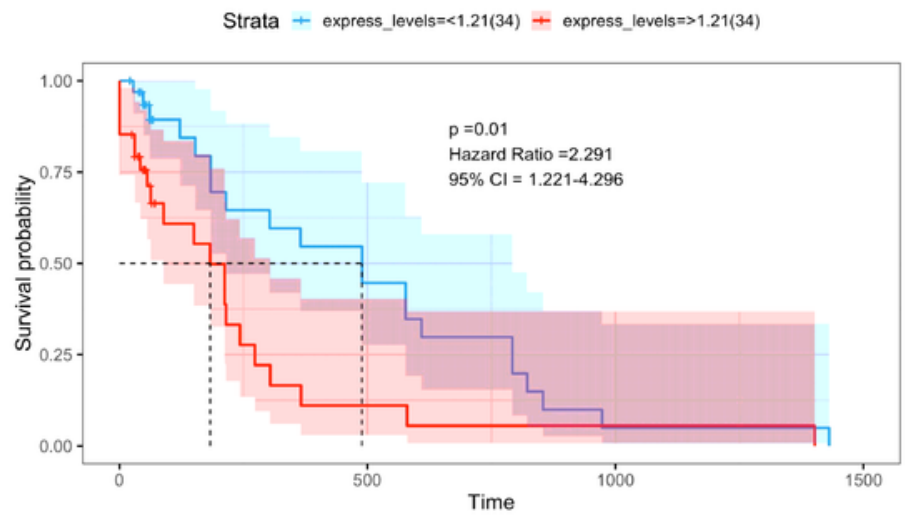

(a) ENSG00000257769 (CTB-193M12.1)

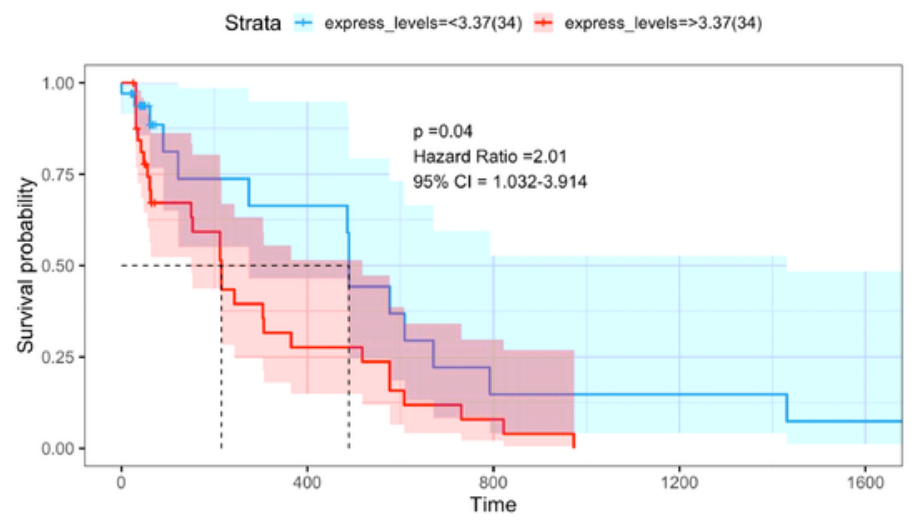

(c) ENSG00000248734 (CTD-2260A17.1)

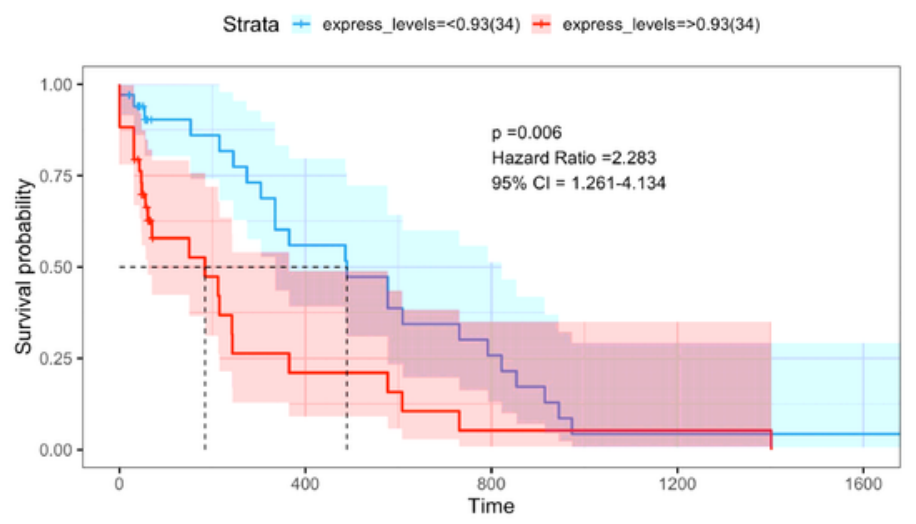

(e) ENSG00000227409 (ZMYM4-AS1)

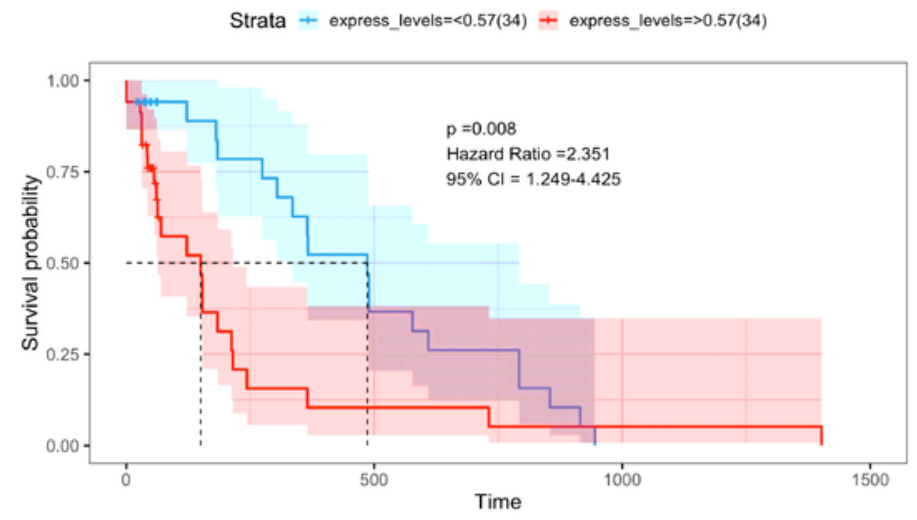

(b) ENSG00000253539 (RP11-402L5.1)

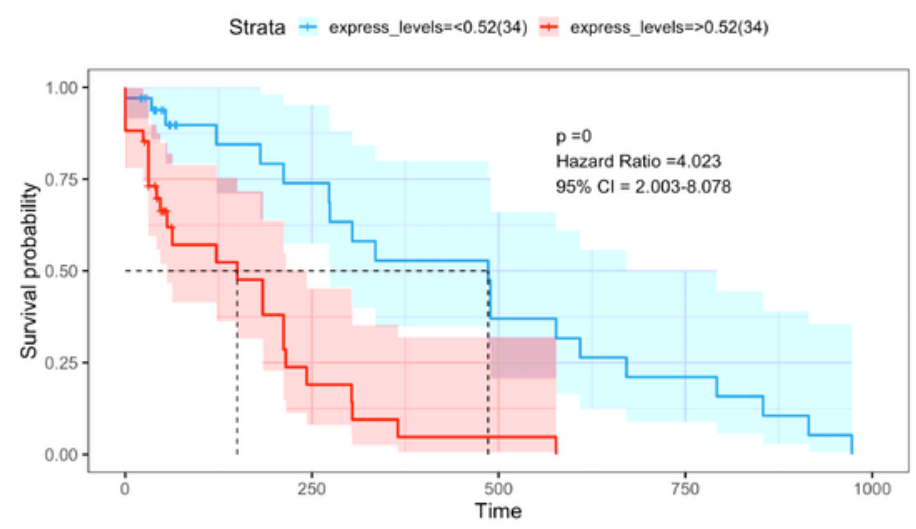

(d) ENSG00000242767 (ZBTB20-AS4)

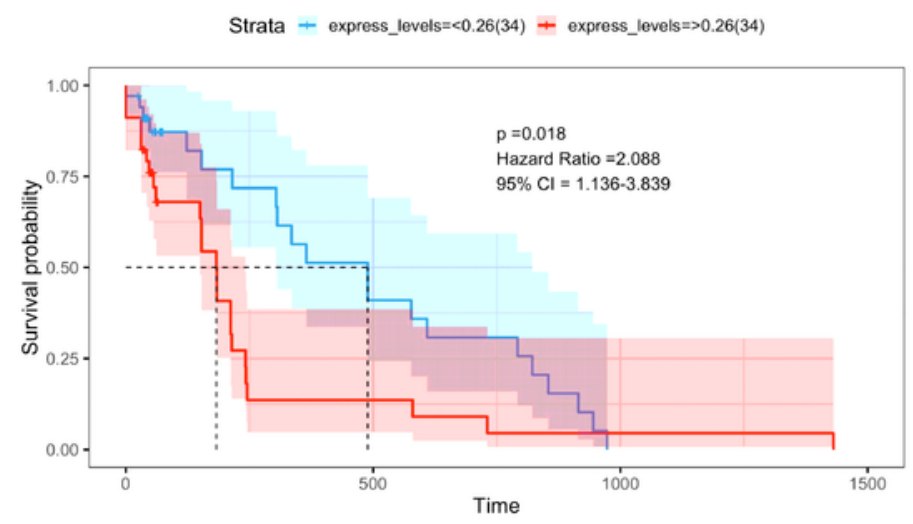

(f) ENSG00000232712 (KIZ-AS1)

\section{Figure 7}

Survival curves of differentially expressed IncRNAs which significantly correlated with overall survival in the regulatory network of ceRNA. 


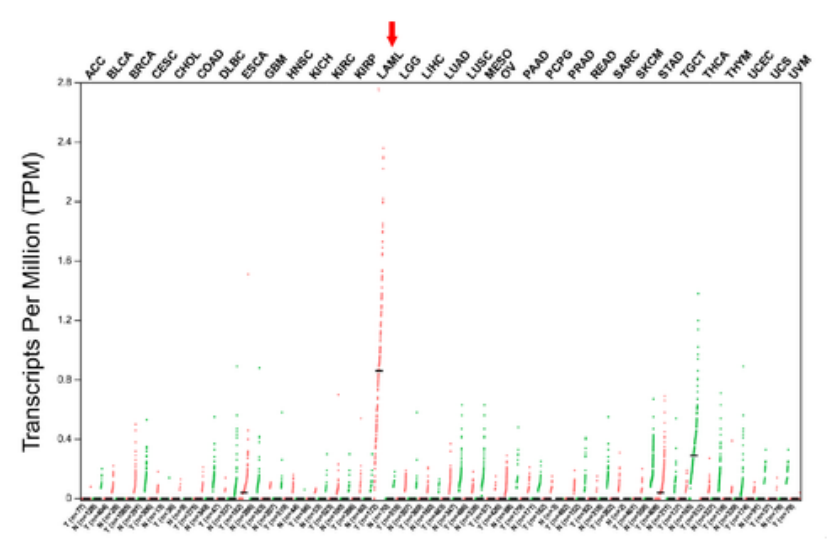

(a)

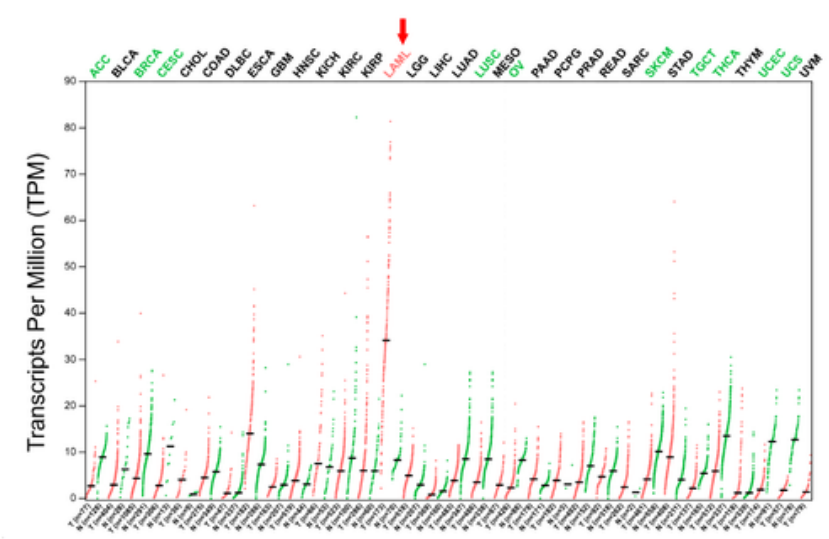

(b)

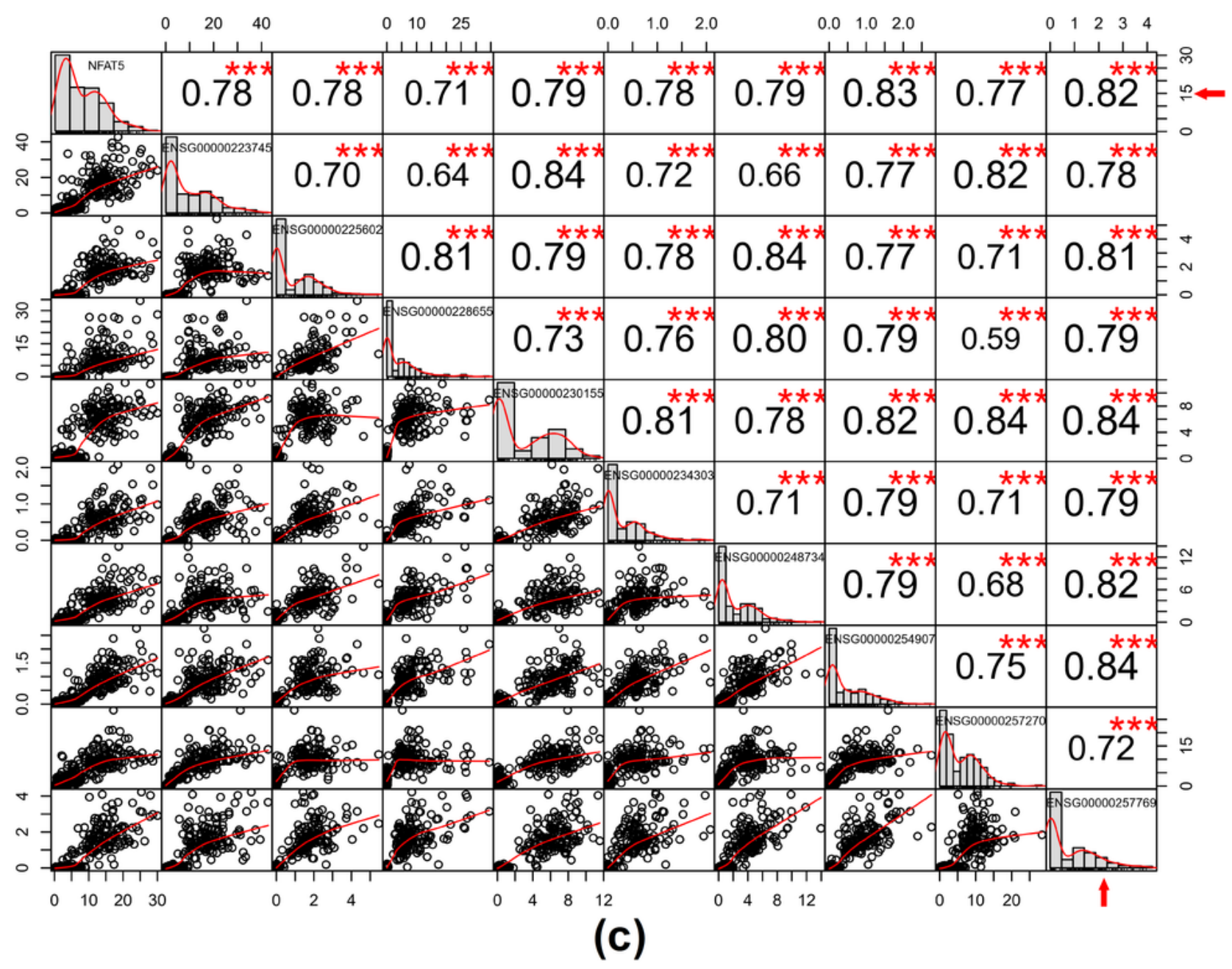

Figure 8

Gene expression and its correlation with the potential regulatory axis of the ceRNA network. (a) CTB193M12.1 is a gene expressed specifically in AML patients of 31 types of tumors; (b) Expression of NFAT5 in 31 types of tumors; (c) Correlation between NFAT5 and 9 IncRNAs, including ENSG00000257769 (CTB-193M12.1). 


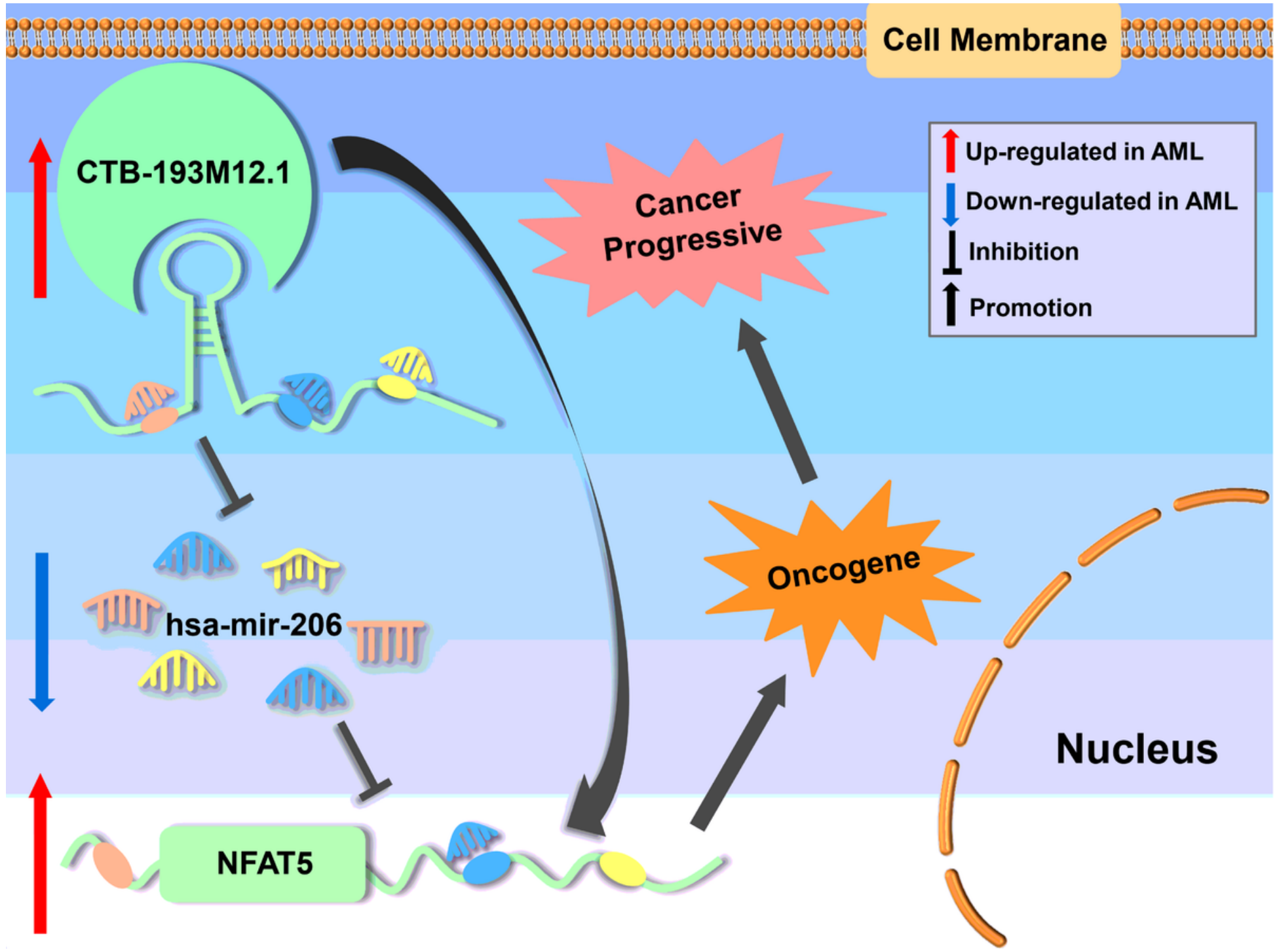

Figure 9

The regulatory axis CTB-193M12.1/hsa-mir-206/NFAT5, which consistent with the ceRNA hypothesis. 


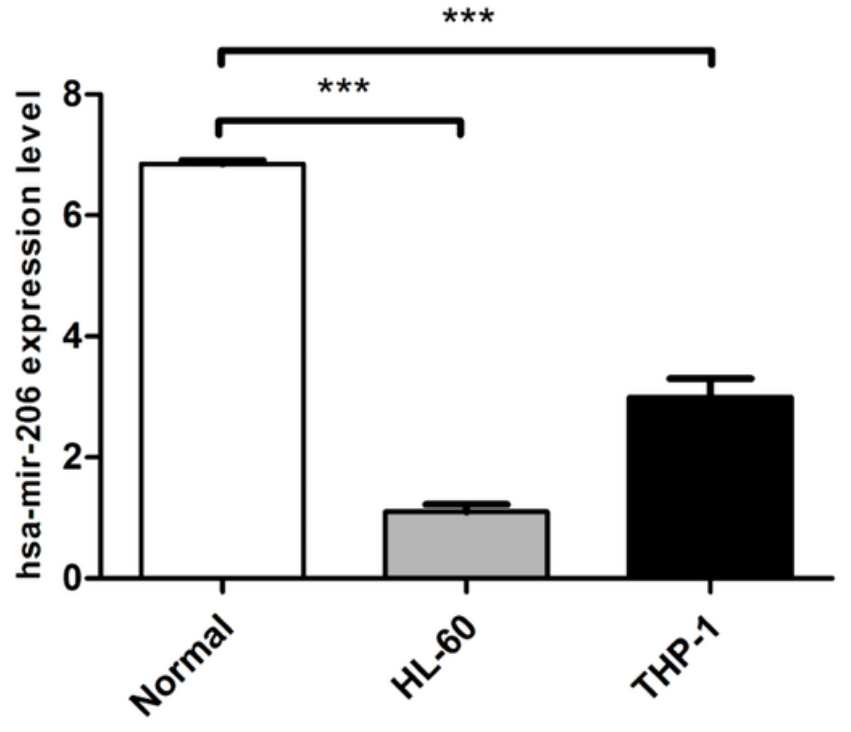

(a)

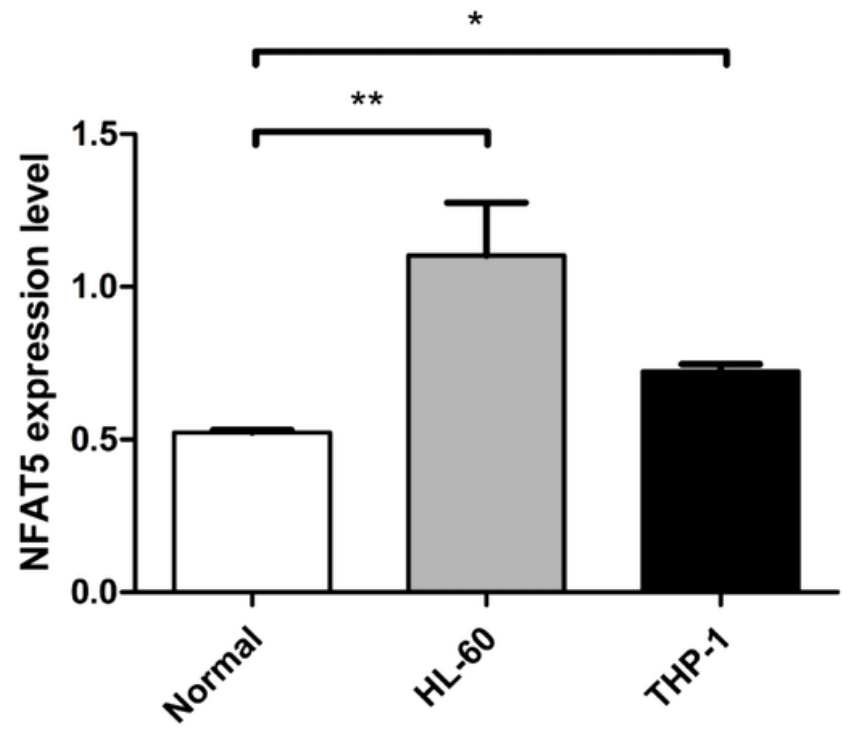

(b)

Figure 10

The hsa-mir-206 and NFAT5 expression in normal human PBMCs, HL-60 cells and THP-1 cells was assayed by qRT-PCR. (a) The hsa-mir-206 expression in normal human PBMCs, HL-60 cells and THP-1 cells; (b) The NFAT5 expression in normal human PBMCs, HL-60 cells and THP-1 cells. ${ }^{*} P<0.05, * * \mathrm{P}<$ $0.01,{ }^{* *} \mathrm{P}<0.001$. 